\title{
Some Discrete Inequalities
}

\author{
S. Varošanec, J. Pečarić and J. Šunde
}

\begin{abstract}
A number of inequalities with finite differences which are connected with weighted, quasiarithmetic and logarithmic means and some well-known general inequalities are considered.

Keywords: Bellman inequality, Cebys̈ev inequality, Hölder inequality, Jensen inequality, Minkowski inequality, Popoviciu inequality, weighted mean, quasiarithmetic mean, logarithmic mean
\end{abstract}

AMS subject classification: $26 \mathrm{D} 15$

\section{Introduction}

In [3] one can find the following generalization of the so-called Pólya inequality (see [3] and [5: Vol. I/p. 57 and Vol. II/p. 114]) and of a result of Balzer (see [1]).

Theorem A. Let $x_{i}:[a, b] \rightarrow \mathbb{R}(1 \leq i \leq n)$ be non-negative increasing functions with continuous first derivative, $p_{i}(1 \leq i \leq n)$ real positive numbers with $\sum_{i=1}^{n} p_{i}=1$ and $f:[a, b] \rightarrow \mathbb{R}$ a non-negative function.

a) If $f$ is non-decreasing, then

$$
\int_{a}^{b}\left(\prod_{i=1}^{n}\left(x_{i}(t)\right)^{p_{i}}\right)^{\prime} f(t) d t \geq \prod_{i=1}^{n}\left(\int_{a}^{b} x_{i}^{\prime}(t) f(t) d t\right)^{p_{i}}
$$

is valid.

b) If $f$ is non-increasing and $x_{i}(a)=0(1 \leq i \leq n)$, then in (1) the reverse inequality is valid.

In this paper we give a discrete: analogue of inequality (1) and some other discrete results.

S. Varoṡanec: Univ. Zagreb, Dept. Math., Bijenička cesta 30, 10000 Zagreb, Croatia

J. Peċarić: Univ. Zagreb, Fac. Textile Techn., Pierottijeva 6, 10000 Zagreb, Croatia

J. Sunde: Univ. Adelaide, Fac. Math. Comp. Sci., Adelaide, South Australia 5001, Australia 


\section{Main results}

2.1 Results in connection with general inequalities. In the following theorems we state some inequalities for finite differences. It is interesting that every of them have its reverse version. It could be done because in the proof we combine general inequalities such as those of Hölder or Minkowski with their reverses such as those of Popoviciu and Bellman. Generally, the proof is given with all details only for one case and the other ones can be proved analogously using related inequalities.

Theorem 1. Let $w=\left(w_{i}\right)_{1 \leq i \leq n}$ and $a_{j}=\left(a_{j i}\right)_{1 \leq i \leq n}(1 \leq j \leq m)$ be non-negative . $n$-tuples and the sums $\sum_{i=1}^{n-1} w_{i} \Delta a_{j i}(1 \leq j \leq m)$ be non-negative. Further, let $p_{j}(1 \leq$ $j \leq m)$ be real numbers such that $\sum_{j=1}^{m} p_{j}=1$.

a) Let $p_{j} \geq 0(1 \leq j \leq m)$. If $w$ is non-decreasing, then

$$
\sum_{i=1}^{n-1} w_{i} \Delta\left(a_{1 i}^{p_{1}} \cdots a_{m i}^{p_{m}}\right) \geq \prod_{j=1}^{m}\left(\sum_{i=1}^{n-1} w_{i} \Delta a_{j i}\right)^{p_{j}}
$$

is valid where $\Delta a_{j i}=a_{j(i+1)}-a_{j i}$ and $\Delta\left(a_{1 i}^{p_{1}} \cdots a_{m i}^{p_{m}}\right)=a_{1(i+1)}^{p_{1}} \cdots a_{m(i+1)}^{p_{m}}-a_{1 i}^{p_{1}} \cdots a_{m i}^{p_{m}}$. If $w$ is non-increasing and $a_{j 1}=0(1 \leq j \leq m)$, then in (2) the reverse inequality is valid.

b) Let $p_{1}>0$ and $p_{j}<0(2 \leq j \leq m)$. If $w$ is non-increasing and $a_{j 1}=0(1 \leq$ $j \leq m)$, then $(2)$ is valid.

Proof. First, let us recall Popoviciu's inequality (see [2: p. 118]): Let $w=$ $\left(w_{i}\right)_{1 \leq i \leq n}$ and $a_{j}=\left(a_{j i}\right)_{1 \leq i \leq n}(1 \leq j \leq m)$ be non-negative $n$-tuples such that

$$
w_{1} a_{j 1}-w_{2} a_{j 2}-\ldots-w_{n} a_{j n} \geq 0 \quad(1 \leq j \leq m)
$$

and let $p_{j}(1 \leq j \leq m)$ be real numbers such that $\sum_{j=1}^{m} p_{j}=1$.

(i) If $p_{j}>0(1 \leq j \leq m)$, then

$$
\begin{aligned}
& \prod_{j=1}^{m}\left(w_{1} a_{j 1}-w_{2} a_{j 2}-\ldots-w_{n} a_{j n}\right)^{p_{j}} \\
& \quad \leq w_{1} a_{11}^{p_{1}} a_{21}^{p_{2}} \ldots a_{m 1}^{p_{m}}-w_{2} a_{12}^{p_{1}} a_{22}^{p_{2}} \ldots a_{m 2}^{p_{m}}-\ldots-w_{n} a_{1 n}^{p_{1}} a_{2 n}^{p_{n}} \ldots a_{m n}^{p_{m}}
\end{aligned}
$$

is valid.

(ii) If $p_{1}>0$ and $p_{j}<0(2 \leq j \leq m)$, then in (3) the reverse inequality is valid.

Now, for proving assertion a) define $\Delta w_{i-1}=w_{i}-w_{i-1}$. If $w$ is non-decreasing, then 
$\Delta w_{i-1} \geq 0$ and we have

$$
\begin{aligned}
& \sum_{i=1}^{n-1} w_{i} \Delta\left(a_{1 i}^{p_{1}} \cdots a_{m i}^{p_{m}}\right) \\
& \quad=w_{n} a_{1 n}^{p_{1}} a_{2 n}^{p_{n}} \cdots a_{m n}^{p_{m}}-w_{1} a_{11}^{p_{1}} a_{21}^{p_{2}} \cdots a_{m 1}^{p_{m}}-\sum_{i=2}^{n} a_{i 1}^{p_{1}} a_{i 1}^{p_{2}} \cdots a_{i 1}^{p_{m}} \Delta w_{i-1} \\
& \quad \geq \prod_{j=1}^{m}\left(w_{n} a_{j n}-w_{1} a_{j 1}-\sum_{i=2}^{n} a_{j i} \Delta w_{i-1}\right)^{p_{j}} \\
& \quad=\prod_{j=1}^{m}\left(\sum_{i=1}^{n-1} w_{i} \Delta a_{j i}\right)^{p_{j}}
\end{aligned}
$$

where inequality (3) is used. If $w$ is non-increasing, then the Hölder inequality is used instead that of Popoviciu. The proof of assertion b) is similar to the previous one

Theorem 2. Let $w=\left(w_{i}\right)_{1 \leq i \leq n}$ and $a_{j}=\left(a_{j i}\right)_{1 \leq i \leq n}(1 \leq j \leq m)$ be non-negative $n$-tuples such that for some $p \in \mathbb{R}$ the sums $\sum_{i=1}^{n-1} w_{i} \Delta a_{j i}^{p}(1 \leq j \leq m)$ are non-negative.

a) Let $w$ be non-decreasing. If $p>1$ or $p<0$, then

$$
\sum_{i=1}^{n-1} w_{i} \Delta\left(a_{1 i}+\ldots+a_{m i}\right)^{p} \geq\left(\sum_{j=1}^{m}\left(\sum_{i=1}^{n-1} w_{i} \Delta a_{j i}^{p}\right)^{1 / p}\right)^{p}
$$

is valid. If $0<p<1$, then in (4) the reverse inequality is valid.

b) Let $w$ be non-increasing and $a_{j 1}=0(1 \leq j \leq m)$. If $0<p<1$, then (4) is valid. If $p>1$, then in (4) the reverse inequality is valid.

Proof. For proving assertion a) we will use the same idee as in the previous theorem and the Bellman inequality (see [2: p. 118]): Let $a=\left(a_{i}\right)_{1 \leq i \leq n}$ and $b=\left(b_{i}\right)_{1 \leq i \leq n}$ be two non-negative $n$-tuples such that

$$
a_{1}^{p}-a_{2}^{p}-\ldots-a_{n}^{p} \geq 0 \quad \text { and } \quad b_{1}^{p}-b_{2}^{p}-\ldots-b_{n}^{p} \geq 0
$$

where $p>1$ or $p<0$. Then

$$
\begin{aligned}
\left(\left(a_{1}^{p}-a_{2}^{p}-\ldots-a_{n}^{p}\right)^{1 / p}\right. & \left.+\left(b_{1}^{p}-b_{2}^{p}-\ldots-b_{n}^{p}\right)^{1 / p}\right)^{p} \\
& \leq\left(a_{1}+b_{1}\right)^{p}-\left(a_{2}+b_{2}\right)^{p}-\ldots-\left(a_{n}+b_{n}\right)^{p}
\end{aligned}
$$

is valid. If $0<p<1$, then in (5) the reverse inequality is valid. An analogous formula states for $m$-tuples $a_{j}(1 \leq j \leq m)$. Further, analogously assertion b) can be proved using the Minkowski inequality

Remark 2. An integral version of the previous theorem is given in [6]. 
Theorem 3. Let $g=\left(g_{i}\right)_{1 \leq i \leq n}$ and $h=\left(h_{i}\right)_{1 \leq i \leq n}$ be non-negative and nondecreasing $n$-tuples such that $g_{1}=h_{1}=0$. If $f=\left(f_{i}\right)_{1 \leq i \leq n}$ is a non-negative and non-increasing $n$-tuple with $f_{1} \neq 0$, then

$$
f_{1} \sum_{i=1}^{n-1} f_{i} \Delta\left(g_{i} h_{i}\right) \geq\left(\sum_{i=1}^{n-1} f_{i} \Delta g_{i}\right)\left(\sum_{i=1}^{n-1} f_{i} \Delta h_{i}\right)
$$

is valid.

Proof. Using the Čebyšev inequality we obtain

$$
\begin{aligned}
\sum_{i=1}^{n-1} f_{i} \Delta\left(g_{i} h_{i}\right)= & f_{n} g_{n} h_{n}-\sum_{i=2}^{n} g_{i} h_{i} \Delta f_{i-1} \\
= & f_{n} g_{n} h_{n}+\sum_{i=2}^{n} g_{i} h_{i} \Delta \overline{f_{i-1}} \\
\geq & \frac{1}{f_{n}+\sum_{i=2}^{n} \Delta \overline{f_{i-1}}} \\
& \times\left(f_{n} g_{n}+\sum_{i=2}^{n} g_{i} \Delta \overline{f_{i-1}}\right)\left(f_{n} h_{n}+\sum_{i=2}^{n} h_{i} \Delta \overline{f_{i-1}}\right) \\
= & \frac{1}{f_{1}}\left(\sum_{i=1}^{n-1} f_{i} \Delta g_{i}\right)\left(\sum_{i=1}^{n-1} f_{i} \Delta h_{i}\right)
\end{aligned}
$$

where $\overline{f_{i}}=-f_{i}$

2.2 Results in connection with weighted, quasiarithmetic and logarithmic means. The previous results are connected with general inequalities as of Hölder, Minkowski and Cebyšev and their reverse versions. In the following theorem we deal with weighted mean. So, let us recall the definition of that mean.

Definition 1. Let $a=\left(a_{i}\right)_{1 \leq i \leq n}$ and $p=\left(p_{i}\right)_{1 \leq i \leq n}$ be positive $n$-tuples, $\sum_{1=i}^{n} p_{i}=$ 1 and $r \in \mathbb{R}$. Then $M_{p}^{[r]}(a)$ defined by

$$
M_{p}^{|r|}(a)= \begin{cases}\left(\sum_{i=1}^{n} p_{i} a_{i}^{r}\right)^{1 / r} & \text { for } r \neq 0 \\ \prod_{i=1}^{n} a_{i}^{p_{i}} & \text { for } r=0\end{cases}
$$

is the weighted mean of order $r$ of $a$ with weight $p$.

Theorem 4. Let $a=\left(a_{i}\right)_{1 \leq i \leq n}$ and $b=\left(b_{i}\right)_{1 \leq i \leq n}$ be non-negative and nondecreasing $n$-tuples such that $a_{1}=b_{1}$ and $a_{n}=b_{n}$, let $p_{1}$ and $p_{2}$ be positive real numbers such that $p_{1}+p_{2}=1$, and let $r$ and $s$ be arbitrary real numbers. Further, let $f=\left(f_{i}\right)_{1 \leq i \leq n}$ be a non-negative $n$-tuple.

a) Let $f$ be non-decreasing. If $r, s<1$, then

$$
\sum_{i=1}^{n-1} \Delta M_{p}^{[r]}\left(a_{i}, b_{i}\right) f_{i} \geq M_{p}^{[s]}\left(\sum_{i=1}^{n-1} f_{i} \Delta a_{i}, \sum_{i=1}^{n-1} f_{i} \Delta b_{i}\right)
$$


is valid. If $r, s>1$, then in (7) the reverse inequality is valid.

b) Let $f$ be non-increasing. If $r<1<s$, then (7) is valid. If $r>1>s$, then in (7) the reverse inequality is valid.

Proof. For proving assertion a) let us suppose that $r, s<1$. Using the inequality between means we obtain

$$
\begin{aligned}
& M_{p}^{[s]}\left(\sum_{i=1}^{n-1} f_{i} \Delta a_{i}, \sum_{i=1}^{n-1} f_{i} \Delta b_{i}\right) \\
& \leq M_{p}^{[1]}\left(\sum_{i=1}^{n-1} f_{i} \Delta a_{i}, \sum_{i=1}^{n-1} f_{i} \Delta b_{i}\right) \\
&=\sum_{i=1}^{n-1}\left(p_{1} \Delta a_{i}+p_{2} \Delta b_{i}\right) f_{i} \\
&=f_{n} M_{p}^{[1]}\left(a_{n}, b_{n}\right)-f_{1} M_{p}^{[1]}\left(a_{1}, b_{1}\right)-\sum_{i=2}^{n} M_{p}^{[1]}\left(a_{i}, b_{i}\right) \Delta f_{i} \\
& \leq f_{n} M_{p}^{[1]}\left(a_{n}, b_{n}\right)-f_{1} M_{p}^{[1]}\left(a_{1}, b_{1}\right)-\sum_{i=2}^{n} M_{p}^{[r]}\left(a_{i}, b_{i}\right) \Delta f_{i} \\
&= f_{n} M_{p}^{[1]}\left(a_{n}, b_{n}\right)-f_{1} M_{p}^{[1]}\left(a_{1}, b_{1}\right) \\
&-\left(f_{n} M_{p}^{[r]}\left(a_{n}, b_{n}\right)-f_{1} M_{p}^{[r]}\left(a_{1}, b_{1}\right)-\sum_{i=1}^{n-1} \Delta M_{p}^{[r]}\left(a_{i}, b_{i}\right) f_{i}\right) \\
&= \sum_{i=1}^{n-1} \Delta M_{p}^{[r]}\left(a_{i}, b_{i}\right) f_{i} .
\end{aligned}
$$

which is the first assertion. The other cases can be proved analogously using the inequality between means

Definition 2. Let $f:[a, b] \rightarrow \mathbb{R}$ be a monotone function with inverse $f^{-1}$, and let $p=\left(p_{i}\right)_{1 \leq i \leq n}$ and $a=\left(a_{i}\right)_{1 \leq i \leq n}$ be real $n$-tuples. Then $M_{f}(a ; p)$ defined by

$$
M_{f}(a ; p)=f^{-1}\left(\frac{1}{P_{n}} \sum_{i=1}^{n} p_{i} f\left(a_{i}\right)\right)
$$

with $P_{n}=\sum_{i=1}^{n} p_{i}$ is the quasiarithmetic $f$-mean of $a$ with weight $p$.

If $p$ is non-negative, $P_{n}=1$ and $f(x)=x^{r} \quad(r \neq 0)$ or $f(x)=\ln x$, then the quasiarithmetic mean $M_{f}(a ; p)$ is the weighted mean $r M_{p}^{[r]}(a)$ of order $r$.

Theorem 5. Let $p=\left(p_{i}\right)_{1 \leq i \leq n}$ be a positive $n$-tuple, $x_{i}=\left(x_{i j}\right)_{1 \leq j \leq m}(1 \leq i \leq n)$ non-negative $m$-tuples with $x_{i^{\prime} 1}=x_{i^{\prime \prime} 1}$ and $x_{i^{\prime} m}=x_{i^{\prime \prime} m}$ for $1 \leq i^{\prime}, i^{\prime \prime} \leq n$, and $w=$ $\left(w_{j}\right)_{1 \leq j \leq m}$ a non-negative $m$-tuple. Further, let $f$ and $g$ be real functions and suppose that all quasiarithmetic means below are well defined. 

then

a) Let $w$ be non-decreasing. If $f$ and $g$ are convex increasing or concave decreasing,

$$
M_{f}\left(\left(\sum_{k=1}^{m-1} w_{k} \Delta x_{i k}\right)_{i} ; p\right) \geq \sum_{k=1}^{m-1} w_{k} \Delta M_{g}\left(\left(x_{i k}\right)_{i} ; p\right)
$$

is valid. If $f$ and $g$ are concave increasing or convex decreasing, then in (8) the reverse inequality is valid.

b) Let $w$ be non-increasing. If $f$ is convex increasing or concave decreasing and $g$ concave increasing or convex decreasing, then (8) is valid. If $f$ is concave increasing or convex decreasing and $g$ convex increasing or concave decreasing, then in (8) the reverse inequality is valid.

Proof. Let us suppose that $f$ and $g$ are convex increasing. We will use the wellknown Jensen inequality, namely, if $0<p_{i} \in \mathbb{R}$ and $x_{i} \in[a, b](1 \leq i \leq n)$ are such that $\frac{1}{P_{n}} \sum_{i=1}^{n} p_{i} x_{i} \in[a, b]$, then for every convex function $f:[a, b] \rightarrow \mathbb{R}$ we have

$$
f\left(\frac{1}{P_{n}} \sum_{i=1}^{n} p_{i} x_{i}\right) \leq \frac{1}{P_{n}} \sum_{i=1}^{n} p_{i} f\left(x_{i}\right)
$$

where $P_{n}=\sum_{i=1}^{n} p_{i}$. So, we obtain

$$
\begin{aligned}
M_{f}\left(\left(\sum_{k=1}^{m-1} w_{k} \Delta x_{i k}\right)_{i} p\right) \\
=f^{-1}\left(\frac{1}{P_{n}} \sum_{i=1}^{n} p_{i} f\left(\sum_{k=1}^{m-1} w_{k} \Delta x_{i k}\right)\right) \\
\geq \frac{1}{P_{n}} \sum_{i=1}^{n} p_{i} \sum_{k=1}^{m-1} w_{k} \Delta x_{i k} \\
=\sum_{k=1}^{m-1} \frac{1}{P_{n}}\left(\sum_{i=1}^{n} p_{i} \Delta x_{i k}\right) w_{k} \\
=\frac{1}{P_{n}}\left(\sum_{i=1}^{n} p_{i} x_{i m}\right) w_{m}-\frac{1}{P_{n}}\left(\sum_{i=1}^{n} p_{i} x_{i 1}\right) w_{1} \\
\quad-\sum_{k=2}^{m} \frac{1}{P_{n}}\left(\sum_{i=1}^{n} p_{i} x_{i k}\right) \Delta w_{k} \\
\geq \frac{1}{P_{n}}\left(\sum_{i=1}^{n} p_{i} x_{i m}\right) w_{m}-\frac{1}{P_{n}}\left(\sum_{i=1}^{n} p_{i} x_{i 1}\right) w_{1} \\
\quad-\sum_{k=2}^{m} g^{-1}\left(\frac{1}{P_{n}}\left(\sum_{i=1}^{n} p_{i} g\left(x_{i k}\right)\right) \Delta^{\Delta} w_{k}\right.
\end{aligned}
$$




$$
\begin{aligned}
& =\sum_{k=1}^{m-1} \Delta g^{-1}\left(\frac{1}{P_{n}}\left(\sum_{i=1}^{n} p_{i} g\left(x_{i k}\right)\right)\right) w_{k} \\
& =\sum_{k=1}^{m-1} w_{k} \Delta M_{g}\left(\left(x_{i k}\right)_{i} ; p\right)
\end{aligned}
$$

which is the first assertion. The other cases can be proved analogously

Remark 3. If $p_{1}>0$ and $p_{i}<0(2 \leq i \leq n)$, then using the reverse version of the Jensen inequality we can state similar results as in the previous theorem. For another weaker condition on $p$ see $[2:$ p. 6].

Definition 3. Let us define the logarithmic mean $L_{r}(x, y)$ by

$$
L_{r}(x, y)= \begin{cases}\left(\frac{1}{y-x} \frac{y^{r+1}-x^{r+1}}{r+1}\right)^{1 / r} & \text { for } r \neq-1,0 \\ \frac{1}{e}\left(\frac{y^{y}}{x^{x}}\right)^{1 /(y-x)} & \text { for } r=0 \\ \frac{y-x}{\ln y-\ln x} & \text { for } r=-1\end{cases}
$$

if $x>0$ and $y>0$ are such that $x \neq y$, and by $L_{\mathbf{r}}(x, x)=x$ (see [2: p. 41]).

Theorem 6. Let $a=\left(a_{i}\right)_{1 \leq i \leq n}$ and $b=\left(b_{i}\right)_{1 \leq i \leq n}$ be non-negative and nondecreasing $n$-tuples such that $a_{1}=b_{1}$ and $a_{n}=b_{n}$, and $w=\left(w_{i}\right)_{1 \leq i \leq n}$ a non-negative $n$-tuple. Further, let $r$ and $s$ be real numbers.

a) Let $w$ be non-decreasing. If $r, s \leq 1$, then

$$
L_{r}\left(\sum_{j=1}^{n-1} w_{j} \Delta a_{j}, \sum_{j=1}^{n-1} w_{j} \Delta b_{j}\right) \leq \sum_{j=1}^{n-1} w_{j} \Delta L_{s}\left(a_{j}, b_{j}\right)
$$

is valid. If $r, s \geq 1$, then in (9) the reverse inequality is valid.

b) Let $w$ be non-increasing. If $r<1<s$, then (9) is valid. If $r>1>s$, then in (9) the reverse inequality is valid.

Theorem 6 can be proved using the inequality for logarithmic mean, i.e. $L_{r}(x, y) \leq$ $L_{9}(x, y)$ for $r \leq s$.

Remark 4. An integral version of Theorems $4-6$ is given in [4]. 


\section{References}

[1] Alzer, H.: An extension of an inequality of G. Pólya. Bul. Inst. Polit. Din Iạsi 36 (1990), $17-18$.

[2] Mitrinović, D. S., Pećarić, J. E. and A. M. Fink: Classical and New Inequalities in Analysis. Dortrecht: Kluwer Acad. Publ. 1993.

[3] Pečarić, J. E. and S. Varošanec: A generalization of Pólya's inequalities. In: Inequalities and Applications. Singapore: World Sci. Publ. Comp. Vol. 3, 1994, pp. $501-504$.

[4] Pečarić, J., Sunde, J. and S. Varošanec: On Gauss-Polya's inequality. Manuscript.

[5] Pólya, G. and G. Szegö: Aufgaben und Lehrsätze aus der Analysis. Vol. I and II., 2nd ed. Berlin: Springer-Verlag 1954.

[6] Varošanec, S.: Inequalities of Minkowski's type. Real Analysis Exchange 20 (1994 - 95), $250-255$.

Received 02.01.1996 